\title{
Second and subsequent fragments headers compression scheme for IPv6 header in 6LoWPAN network
}

\begin{abstract}
Wireless Embedded Internet aims for efficient connectivity for embedded devices to the internet. This requires the embedded devices to run IPv6 protocol. The 6LoWPAN was introduced to enable IPv6 internet connectivity for WPAN. Enabling IPv6 in wireless, small size, low power, low rate, limited memory and limited computation capabilities devices, with a limited frame size, is not directly applicable. The relatively huge header size of upper layers' protocols (e.g. TCP, UDP and IPv6), in addition to IEEE 802.15.4 header, will deplete the frame payload to approximately 33 bytes. Some schemes had been designed to compress the headers to provide more space for the data payload. In this paper, we present a header compression scheme for 6LoWPAN network. The scheme exploits the correlation between the first and the subsequent fragments' headers. Hence, the redundant headers that are transmitted within the first fragment will not be carried again within the second and the subsequent fragments. Second and Subsequent Fragments Headers Compression Scheme (S\&SFHC) can either work as a standalone technique or be integrated with other compassion techniques. However, in this paper, we assess the standalone scheme where the scheme is not integrated with other compression scheme. The performance of the S\&SFHC is evaluated based on packet delivery ratio, total charged consumed, average throughput and average delay. It achieved $20 \%, 6 \%, 26 \%$ and $6 \%$ better performance in terms of packet delivery ratio, total charged consumed, average throughput and average delay compared to LOWPAN_IPHC as increasing the size of fragmented packets.
\end{abstract}

Keyword: 6LoWPAN; Header compression; IPv6; IEEE 802.15.4 